\title{
3D VASCULAR PATH PLANNING OF CHEMO-EMBOLIZATIONS USING SEGMENTED HEPATIC ARTERIES FROM MR ANGIOGRAPHY
}

\author{
Anaïs Badoual ${ }^{\star 1}$, Maxime Gerard ${ }^{\star \dagger}$, Benjamin de Leener ${ }^{\dagger}$, Nadine Abi-Jaoudeh $^{*}$,Samuel Kadoury ${ }^{\star \dagger}$ \\ ${ }^{\star}$ MedICAL, Ecole Polytechnique Montréal, Montreal, QC, Canada \\ ${ }^{*}$ Rad. and Imaging Sciences, National Institutes of Health, Bethesda, MD, USA \\ ${ }^{\dagger}$ Institute of Biomedical Engineering, Polytechnique Montréal, Montreal, QC, Canada \\ ${ }^{1}$ Biomedical Imaging Group, Ecole Polytechnique Fédérale de Lausanne, Switzerland
}

\begin{abstract}
Transcatheter arterial chemo-embolization (TACE) is a therapeutic procedure to treat primary and metastatic liver cancer. It requires prior delineation of the hepatic arteries on magnetic resonance angiography (MRA) data and identification of the vessels supplying the tumor. Manual segmentation is extremely challenging and time consuming, thereby increasing the risk of wrongfully identifying the feeding vessels. We present a vascular path planning tool for TACE procedures by automatically segmenting the hepatic arteries on MRA. The proposed method first detects the celiac trunk from the aorta, then localizes and tags bifurcations throughout the arterial network for path planning. The algorithm is based on a multiple hypothesis tracking approach used to propagate deformable mesh surfaces. We validated the proposed framework on 20 liver-cancerpatients using abdominal MRA with 20 seconds delay after contrast injection. We show that the algorithm improves the selectivity of the arterial segments and outperforms two state-of-the-art methods with respect to manual segmentation, yielding mesh geometries with a mean absolute distance of $0.61 \pm 0.10 \mathrm{~mm}$ for 3D mesh models.
\end{abstract}

Index Terms - Liver cancer, transcatheter arterial chemoembolization, vascular path planning, hepatic artery segmentation, magnetic resonance angiography.

\section{INTRODUCTION}

Primary liver tumor known as hepatocellular carcinoma (HCC) is diagnosed in more than half a million people worldwide per year [1]. Transcatheter arterial chemo-embolization (TACE) is the preferred therapeutic approach for treating advanced HCC. Thereby, a catheter is inserted into a branch of the hepatic artery that supplies the tumor. A chemotherapeutic mixture followed by (drug-eluding) beads is injected into the arterioles supplying the tumor. Determining these arterioles is vital to minimize damage of normal liver tissue and increase the embolic effect and chemotherapeutic concentration in the tumor. Vascular path planning enables the clinician to determine the hepatic branches that supply the tumor, as well as to plan the vascular route of the catheter, starting from the celiac artery and leading to the subsegmental branch artery. Most clinical procedures rely on visual determination of the blood supply based on 2D angiography which is difficult and imprecise. The amount of data generated from 3D angiographic imaging modalities, such as MRA makes the manual segmentation task tedious and challenging. (Semi-)automatic methods isolating the vascular network simplify this step for clinicians by

This work was supported by the Canada Research Chairs and the Canadian Institutes of Health Research (CIHR). minimizing manual interaction and reducing inter-operator variability in order to visualize the hepatic arteries.

Due to the hepatic vascular network's complex structure, presenting high variability with respect to size and curvature, in addition to resolution difference, noise artefacts and injection variations, extracting liver vasculature in MRA remains challenging. Methods based on region growing, active contours, tracking or deformable models [2][3] ave been proposed to segment the coronary [4] or cerebral vessels [5]. However these approaches are difficult to adapt to hepatic arteries due to challenges mentioned above. Particularly in the liver, the contrast is low and arteries are small and surrounded by tubular structures which are the main difficulties when segmenting these arteries. Few methods have been applied to segment the liver arteries. Existing approaches are often either sensitive to small radii [6] or they only segment partially the hepatic arteries [7]. To the best of our knowledge, no framework enables the extraction of hepatic arteries in MRA images for 3D roadmapping.

We present a path planning approach for arterial procedures based on the propagation of deformable models in MRA volumes (after contrast agent injection to enhance blood vessels). The algorithm detects and localizes bifurcations throughout the vascular network which is a crucial step [8] in this context. Segmentation of the vascular tree is carried out up to the third level of bifurcation, where the catheter is typically guided in TACE procedures. Our contributions are 1) a novel automatic pre-segmentation step that detects the celiac trunk, i.e., the seeding point of the hepatic tree whose proper initialization is key for an accurate segmentation outcome; 2) a vessel extraction step by combining an existing model to track the vessel path [7] and an extension of the mesh propagation model presented in [9] to segment the vessel walls; 3) the generation of the hepatic network as a 3D mesh and the complete characterization of the bifurcations (coordinates and level) as well as of the paths; these contributions are at the core of the proposed framework. Validation with respect to two state of the art methods show high accuracy in $3 \mathrm{D}$ vascular maps and low rates in terms of detection errors.

\section{METHODS}

The proposed vascular path delineation relies on a two-step approach. First, both the aorta and the celiac trunk are automatically detected and segmented on the vesselness image (Sec. 2.1), where the root of the hepatic artery originates from the aorta. This provides a seed point and a direction to initialize the extraction of the hepatic arteries. The segmentation step consists in the tracking of the vessel paths (Sec. 2.2) and the propagation of deformable models to segment the vessel walls (Sec. 2.3). 


\subsection{Aorta and celiac trunk segmentation}

In this section we present an iterative propagation routine of tubular deformable mesh models based on [9]. In order to adapt for the segmentation of the aorta, the length and radius of the structure of interest is configured. An elliptical Hough transform is applied to the medial plane of several adjacent slices to detect the starting point in the aorta. It is performed on the vesselness image of the MRA by applying the filter to enhance tubular structures and reduce noise due to surrounding structures within the image.

Using the resulting position and direction an initial (regular) cylindrical triangular mesh is built. It is then deformed by minimizing the energy (1) which attracts the model towards the edges of the aorta. It is given by

$$
\begin{aligned}
E(\mathbf{v})= & \sum_{i=1}^{T}\left(\frac{g\left(\tilde{\mathbf{x}}_{i}\right)}{\left\|g\left(\tilde{\mathbf{x}}_{i}\right)\right\|}\left(\tilde{\mathbf{x}}_{i}-\mathbf{x}_{i}\right)\right)^{2}+ \\
& \alpha \sum_{j=1}^{V} \sum_{k \in N(j)}\left(\mathbf{v}_{j}-\mathbf{v}_{k}-\mathbf{R}\left(\mathbf{v}_{j}^{1}-\mathbf{v}_{k}^{1}\right)\right)^{2}
\end{aligned}
$$

where $\|g\|$ is the gradient magnitude of the image, $V$ and $T$ are the number of vertices and triangles respectively, $\left\{\mathbf{x}_{i}\right\}_{i \in[1, T]}$ are the centroids of the triangles, $\mathbf{v}$ and $\mathbf{v}^{1}$ are the vertices of the current and the initial mesh respectively, $N(j)$ the neighborhood of vertex $j$ and $\mathbf{R}$ the rotation matrix aligning the initial and the current mesh. The first term in (1) is the external energy which updates the centroids of the triangles towards their most promising locations $\left\{\tilde{\mathbf{x}}_{i}\right\}_{i \in[1, T]}$ defined by $\tilde{\mathbf{x}}_{i}=\mathbf{x}_{i}+k h \mathbf{n}_{i}$, where:

$$
k=\underset{j=-l, \ldots, l}{\arg \max }\left\{\mathbf{n}_{i} \mathbf{g}\left(\mathbf{x}_{i}+j h \mathbf{n}_{i}\right)-d h^{2} j^{2}\right\},
$$

$2 l+1$ is the size of the neighborhood along the triangle normals, $h$ the distance between two points along this normal, $d$ a trade-off parameter between gradient and distance, and $\mathbf{n}_{i}$ the normal at $\mathbf{x}_{i}$. The second term in (1) is the internal energy which constrains the mesh to preserve topology. The trade-off between mesh flexibility and surface coherence is controlled by $\alpha$. After the initial mesh deformation it is iteratively propagated in two opposite directions in order to segment the walls of the aorta along it's central axis (i.e. up to the level of the celiac trunk). At each iteration an extremity of the current deformed mesh is duplicated and translated in the direction of the structure of interest to become reoriented and deformed towards the aorta walls using (1). Each new orientation of the triangular mesh is computed by maximizing (3), i.e., the image gradient values for all the triangle centroids as

$$
E_{\text {orient }}=\sum_{i=1}^{T}\left\|g\left(\mathbf{R}\left(\mathbf{x}_{i}-\mathbf{p}\right)+\mathbf{p}\right)\right\|,
$$

where the centroids $\left\{\mathbf{x}_{i}\right\}_{i \in[1, T]}$ are rotated by $\mathbf{R}$ about $\mathbf{p}$, which is the center of mass of the lower extremity of the updated mesh. Parameters $\alpha, d$ and $h$ were found empirically using test data different from the validation data.

We detect the bifurcation between the aorta and the celiac trunk by a dual thresholding on the intensity with proximity constraints to the segmented aorta. To eliminate false positives (typically points on the mesenteric artery) a spectral clustering is applied on the 3D coordinates of the remaining points. The cluster corresponding to the celiac trunk has the highest centroid as analyzed along the caudalcranial axis. An initial mesh of the celiac trunk is created which constitutes the starting point of the tracking method for the hepatic artery.

\subsection{Multiple hypothesis tracking of vessel paths}

\subsubsection{Tracking}

Once the celiac trunk is identified branch bifurcations are detected in order to guide the propagation of the segmentation on each branch of the hepatic artery. We apply a multiple hypothesis tracking method similar to the one presented in [7] on the vesselness image to detect all bifurcations in the network using a search tree algorithm. Let $\beta_{t}$ be the point associated to the set of parameters of a segment of the artery at time $t$. We compute successive segments of the artery such that a track ranging from $\beta_{0}$ up to $\beta_{3}$ is created. A set $\left\{\beta_{t+1}^{(i)}\right\}_{i \in I}$ of cardinality $I$ of possible paths of the vessel is subsequently predicted from $\beta_{t}$. These predictions are placed uniformly $\left(20^{\circ}\right.$ spacing) on a sphere segment defined by an angle $\phi=60^{\circ}$ with respect to the direction of the artery at $\beta_{t}$ and a radius equal to 1.5 times the vessel radius at $\beta_{t}$ (Fig. 1(1)). A t-statistic score based on the local image contrast is associated to each prediction. Finally, a pruning threshold (equal to 4) is applied to these scores to remove weak predictions. The remaining $J$ points $\beta_{t+1}^{\left(j^{*}\right)}$ are taken as local assumptions of the path of the artery and new spheres of predictions are created from these points (Fig. 1(2)). After three steps the search tree is built (Figs. 1(3), 1(4)). The created search tree is auto-evaluated in order to choose the preferred direction originating from $\beta_{t}$. The average score of each branch of the tree leading to the desired depth (i.e., $\beta_{t+3}^{(j *)}$ in Fig. 1(4)) is calculated and a final threshold on this score is applied. To enhance robustness we set the final threshold to be twice the pruning threshold. If no leaf remains, tracking of the current artery is terminated. Otherwise, a bifurcation verification is performed on the remaining leaves. For each detected branch the deformable model is oriented ( $c f$. Section 2.3) and propagated towards the leaf showing the highest mean score (Fig. 1(5)). A new search tree is then built from this new point and the procedure is repeated until the vessel curvature is too large or the structures fall outside the vesselness image.

\subsubsection{Bifurcation localization and tagging}

We detect the bifurcations by performing a spectral clustering on the spatial coordinates of the leaves $\beta_{t+3}^{(j *)}$ from the resulting search tree. First, the matrix $\mathbf{S}$, which measures the similarity between leaves $i$ and $j$ is calculated as

$$
\mathbf{S}_{i j}=\mathbf{S}_{j i}=e^{-2 \frac{\left\|\beta_{t+3}^{(i *)}-\beta_{t+3}^{(j *)}\right\|}{r_{i}+r_{j}}},
$$

where $r_{i}$ and $r_{j}$ are the radii of the artery at the corresponding leaves and $\|\cdot\|$ is the Euclidean 2-norm. An eigen-decomposition of the Laplacian matrix $\mathbf{L}=\mathbf{D}-\mathbf{S}$ is computed, where $\mathbf{D}$ is a diagonal matrix defined as $\mathbf{D}_{i j}=\sum_{j} \mathbf{S}_{i j}$. The second smallest eigenvector of $\mathbf{L}$ determines the different clusters. The first cluster is composed of indices exhibiting negative eigenvector values, while the second cluster is composed of indices where all values of the eigenvector are positive. Finally, a threshold on the distance between the centroids $\mathbf{P}_{i}$ of each detected cluster is applied to identify bifurcations such that if $\left\|\mathbf{P}_{1}-\mathbf{P}_{2}\right\|>1.9 \frac{\left(r_{1}+r_{2}\right)}{2}$, we detect a bifurcation. This process yields the coordinates of each bifurcation associated with their respective level in a branch of the hepatic tree.

\subsection{Mesh propagation along vessel paths}

The segmentation of the hepatic walls is achieved using the propagation routine described in Section 2.1 towards the targets identified 


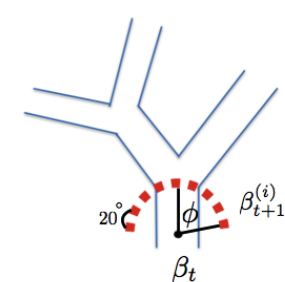

(1) Construction of the first level

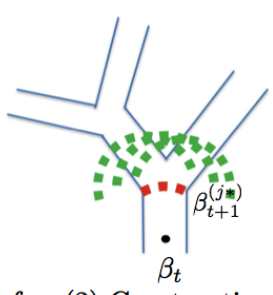

(2) Construction of the second level

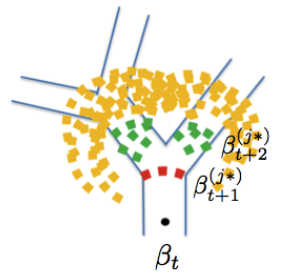

(3) Construction of the third level

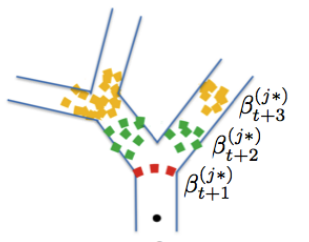

$\beta_{t}$

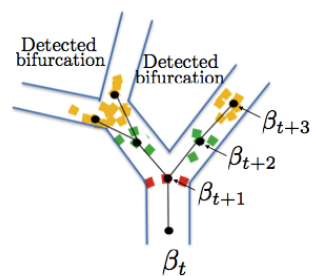

(5) Evaluation
Fig. 1: Search tree construction and evaluation. Blue: hepatic arteries. Red: first level of spherical region of predictions from $\beta_{t}$. Green: second level of spherical region of predictions from $\beta_{t+1}^{(j *)}$. Yellow: third level of spherical region of predictions from $\beta_{t+2}^{(j *)}$. Black tree: branches of the search tree of depth three leading to the identified leaves obtained after evaluation and bifurcation detection.

in the tracking phase. Hepatic arteries are complex structures with different radii, curvatures and bifurcations. The propagation routine needs to be adapted to these challenges. First, the propagation starting from the celiac trunk becomes unidirectional. To compute the length of the duplicated mesh, as well as its new orientation, the curvature of the vessel and the presence of bifurcations have to be taken into account. We propose to compute the propagation routine from the set of points $\left(\beta_{t+1}, \beta_{t+2}\right.$ and $\beta_{t+3}$, Fig. 1(5)) on the branch of the search tree that lead to the detected leaf. At each iteration $i \in[1,3]$ the length of the duplicated mesh is equal to the one of the segment $\left[\beta_{t+i-1} \beta_{t+i}\right]$. Its new orientation is expressed by the angle $\gamma=\arccos \left(\overrightarrow{\beta_{t+i-2} \beta_{t+i-1}}, \overrightarrow{\beta_{t+i-1} \beta_{t+i}}\right)$ (Fig. 2). The propagation stops when the radius is inferior to $0.9 \mathrm{~mm}$ or the maximum number of iterations has been reached $(n=200)$.

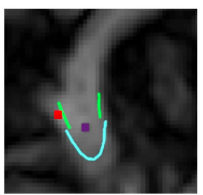

(a) Translation

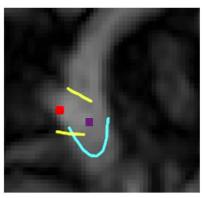

(b) Orientation

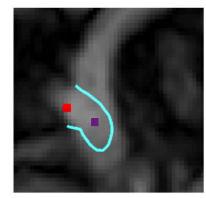

(c) Deformation
Fig. 2: 2D view of segmentation propagation using a search tree. Green: duplicated and translated mesh. Yellow: reoriented duplicated mesh. Blue: mesh after propagation and deformation.

\section{RESULTS AND DISCUSSION}

The method was validated on arterial phase T1-weighted MRA images originating from 20 different patients with primary liver cancer. No pre-selection on image quality was made. They were acquired at breath-hold 15-25 sec. after injection of a Gadolinium- based contrast agent. Image acquisition was performed in the abdominal region with a resolution between $0.7 \times 0.7 \times 2 \mathrm{~mm}^{3}$ and $1.2 \times 1.2 \times 3 \mathrm{~mm}^{3}$. For all patients, the proposed pre-segmentation routine was able to correctly detect the celiac trunk, even with varying anatomies. Its robustness is crucial for the subsequent segmentation process, which takes $10 \mathrm{~min}$ on average on a i5 Intel PC workstation, with 32.0 GByte of memory.

The accuracy of both the extracted vascular paths and segmented meshes were measured. Bifurcation detection was evaluated for segments up to the third level (inclusively) to allow selective embolization during TACE. Segmentation accuracy was compared to a manual segmentation carried out by an expert clinician (gold standard), as well as two commonly used methods for vascular segmentation. The first is based on level-sets with vesselness enhancement, while the second is a recent method using normal and Gumbel intensities distributions for adapted thresholding [10]. For the former method, centrelines were extracted using the method in [11]. These semiautomatic vascular methods are not particularly optimized for hepatic arteries but are comparable to the proposed framework.

\subsection{Vascular path extraction}

In Table 1 accuracy measures of the vascular path extraction are provided. The larger errors in terms of mean absolute distance (MAD) and mean square distance (MSD) obtained with the level-set-based method are mainly due to the non-continuity of the extracted path. One-way Anova tests were performed comparing the averages obtained with the different methods. The corresponding $\mathrm{p}$-values are provided in Table 1.

\begin{tabular}{ccc}
\hline \multicolumn{3}{c}{ 3D Vessel path } \\
\hline & MAD (mm) & MSD (mm) \\
\hline Level-set + vesselness & $1.79 \pm 0.96$ & $11.25 \pm 9.07$ \\
Adaptive thresholding [10] & $1.43 \pm 0.52$ & $3.72 \pm 1.20$ \\
Proposed method & $\mathbf{1 . 0 1} \pm \mathbf{0 . 1 6}$ & $\mathbf{1 . 2 7} \pm \mathbf{0 . 4 4}$ \\
\hline p-value & 0.0081 & 0.0086 \\
\hline
\end{tabular}

Table 1: 3D centreline extraction with the proposed method in comparison with a level-set approach and the adaptive thresholding approach from Gaussian distributions by Wang et al. [10].

Table 2 presents sensitivity, positive predictive value (PPV) and the percentile error for the detection of bifurcation within the vascular tree. The percentile error of bifurcation is defined as \% error $=$ $\frac{M B+F B}{T B}$, where $M B$ and $F B$ are the number of missed bifurcations and false bifurcations obtained with the evaluated method respectively, while $T B$ is the number of true bifurcations identified by a radiologist. We obtain an error rate of $0.77 \%$, which is slightly below the error obtained with the level-set approach with vesselness.

\begin{tabular}{cccc}
\hline \multicolumn{4}{c}{ Bifurcation detection } \\
\hline & Sensitivity & PPV & Error (\%) \\
\hline Level-set + vesselness & $79 \%$ & $67 \%$ & $0.84 \pm 0.81$ \\
Proposed method & $79 \%$ & $\mathbf{6 9} \%$ & $\mathbf{0 . 7 7} \pm \mathbf{0 . 7 4}$ \\
\hline
\end{tabular}

Table 2: Evaluation of the bifurcation detection for the level-set + vesselness and proposed methods compared to the gold standard. 


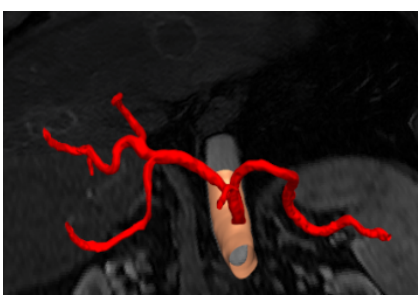

(a)

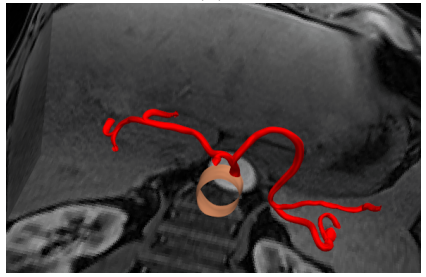

(c)

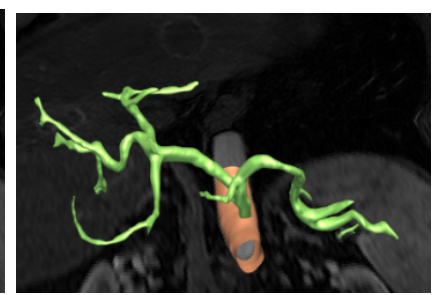

(b)

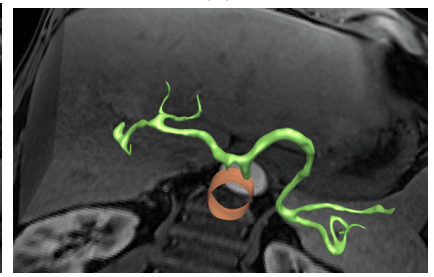

(d)
Fig. 3: (a) and (c) are 3D automatic segmentations; (b) and (d) are the corresponding 3D manual segmentations.

\subsection{D mesh modeling of hepatic arterial walls}

In Table 3 we show the average Hausdorff distance (HD), mean absolute distance (MAD) and mean square distance (MSD) between our segmentation results and the gold standard, as well as between the level-set and adaptive thresholding approaches [10]. The results show that the proposed method yields higher accuracy than both comparative approaches. Furthermore, for the average HD we obtain $3.13 \pm 0.88 \mathrm{~mm}$ which is approximately the radius of the celiac trunk. Our method yields a smooth segmentation, as shown in Fig. 3 with $3 \mathrm{D}$ results compared to manual delineation. In the 2D segmentations showing a comparison between the manual, automatic and the level-set approaches in Fig. 4, the level-set method gives rough meshes with edges and presents discontinuities. Still the large slice distances in the MRA may affect the segmentation of small structures due to partial-volume effects in the image. The p-values from one-way Anova tests are provided in Table 3.

\begin{tabular}{cccc}
\hline \multicolumn{4}{c}{ 3D Mesh Geometry } \\
\hline & MAD (mm) & MSD (mm) & HD (mm) \\
\hline Level-set + vesselness & $2.08 \pm 1.49$ & $20.38 \pm 18.99$ & $15.98 \pm 7.77$ \\
Adaptive thresholding [10] & $1.29 \pm 0.30$ & $3.14 \pm 1.29$ & $6.71 \pm 1.34$ \\
Proposed method & $\mathbf{0 . 6 1} \pm \mathbf{0 . 1 0}$ & $\mathbf{0 . 6 2} \pm \mathbf{0 . 2 4}$ & $\mathbf{3 . 1 3} \pm \mathbf{0 . 8 8}$ \\
\hline p-value & $3.10^{-6}$ & 0.0015 & 0.0210 \\
\hline
\end{tabular}

Table 3: 3D mesh geometry accuracy measures for the proposed automatic method in comparison with the level-set approach and to an adaptive thresholding approach by Wang et al. [10].
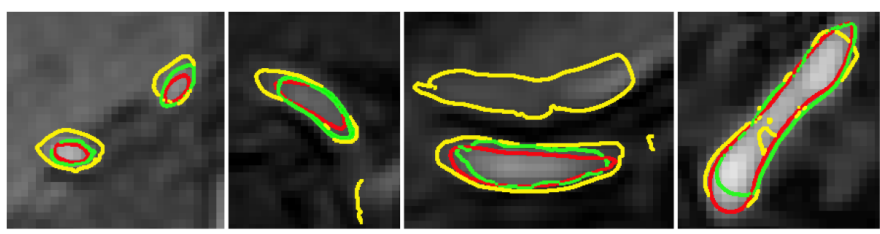

Fig. 4: In-plane segmentation of hepatic arteries. Red: ground-truth; Green: proposed method; Yellow: level-set-based method.

\section{CONCLUSION}

We propose a 3D vascular path planning method which segments the hepatic arteries from MRA in order to assist interventional radiologists in their preoperative planning phase for the treatment of liver cancer. Our main contribution consists in proposing an automatic vascular path planning method designed for MR angiography which detects the celiac trunk and bifucations in the arterial network. The arteries are segmented using deformable vessel models up to the third level of the hepatic tree which is required for guidance in TACE procedures. The proposed method yields higher accuracy for the segmentation of both the vessel path and the 3D mesh model compared to both vesselness and Gaussian methods, while the validation to a gold standard yields low error rates. Additionally, the method also performs well in regions with low contrast and on vessels with small radius. Future work will focus on the computation of additional physiological parameters and a more extensive validation.

\section{REFERENCES}

[1] H.B. El-Serag, "Hepatocellular carcinoma.," New England Journal of Medicine, vol. 365, no. 12, pp. 1118-1127, 2011.

[2] David Lesage, Elsa D. Angelini, Isabelle Bloch, and Gareth Funka-Lea, "A review of $3 \mathrm{~d}$ vessel lumen segmentation techniques: Models, features and extraction schemes.," Medical Image Analysis, vol. 13, no. 6, pp. 819-845, 2009.

[3] C. McIntosh and G. Hamarneh, "Vessel crawlers: 3d physically-based deformable organisms for vasculature segmentation and analysis.," 2006, vol. 1, pp. 1084-1091.

[4] Yefeng Zheng, Hseyin Tek, and Gareth Funka-Lea, "Robust and accurate coronary artery centerline extraction in cta by combining model-driven and data-driven approaches.," in MICCAI (3), 2013, vol. 8151, pp. 74-81.

[5] Albert C. S. Chung, J. Alison Noble, and Paul E. Summers, "Vascular segmentation of phase contrast magnetic resonance angiograms based on statistical mixture modeling and local phase coherence," IEEE Trans. Med. Imaging, vol. 23, no. 12, pp. 1490-1507, 2004.

[6] B.B. Frericks and et al., "3d ct modeling of hepatic vessel architecture and volume calculation in living donated liver transplantation.," European Radiology, vol. 14, no. 2, pp. $326-$ 333, February 2004.

[7] O. Friman, M. Hindennach, C. Khnel, and H.O. Peitgen, "Multiple hypothesis template tracking of small 3D vessel structures," Medical Image Analysis, vol. 14, pp. 160-171, 2010.

[8] Mengliu Zhao and Ghassan Hamarneh, "Bifurcation detection in 3d vascular images using novel features and random forest," in IEEE Int. Symp. on Bio. Im., 2014, pp. 421-424.

[9] B. De Leener, J. Cohen-Adad, and S. Kadoury, "Automatic segmentation of the spinal cord and spinal canal coupled with vertebral labeling," IEEE Trans. Med. Imaging, vol. 34, pp. 1705-18, 2015.

[10] Rui Wang, Chao Li, Jie Wang, et al., "Threshold segmentation algorithm for automatic extraction of cerebral vessels from brain magnetic resonance angiography images," Journal of neuroscience methods, vol. 241, pp. 30-36, 2015.

[11] M. Schaap, C.T. Metz, T. van Walsum, and et al., "Standardized evaluation methodology and reference database for evaluating coronary artery centerline extraction algorithms," Medical Image Analysis, vol. 13/5, pp. 701-714, 2009. 